\title{
Effects of Cannabinoid and Trpv1 in Formalin-induced Orofacial Pain
}

\author{
Min-Kyoung Park ${ }^{1}$, Man Hyu Huh ${ }^{2}$, Min-Kyung Lee ${ }^{3}$ \\ ${ }^{1}$ Assistant Professor, Department Of Dental Hygiene, Kyung-Woon University, Republic Of Korea \\ ${ }^{2}$ Professor, Department Of Molecular Biology, Dong-Eui University, Republic Of Korea \\ ${ }^{3}$ Assistant Professor, Department Of Dental Hygiene, Dong-Eui University, Republic Of Korea
}

\begin{abstract}
This study investigated that the modulation of cannabinoid (CB) or TRPVI (transient receptor potential vanilloid) in inflammatory orofacial pain. Experiments were carried out using adult male SpragueDawley rats weighing 240 to $280 \mathrm{~g}$. Formalin $(5 \%, 30 \mu \mathrm{l})$ were applied subcutaneously to the vibrissa pad. For each animal, the number of noxious behavioral responses, including rubbing or scratching of the facial region proximal to the injection site, was recorded for 9 sequential 5 minute intervals. Anandamide (AEA), a CB1 agonist, was injected subcutaneously on 20 minute prior to formalin injection. The subcutaneous injection from 0.03 to $0.3 \mu \mathrm{g}$ of AEA suppressed the number of scratches in the second phase of the behavioral responses to formalin. Low doses of AEA were more significantly suppressed the number of scratches in the second phase. The subcutaneous injection of $10 \mu \mathrm{g}$ of AM251, a CB1 antagonist, and $0.4 \mu \mathrm{g}$ of IRTX, a TRPV1 antagonist, were injected at 10 minute prior to AEA injection. Pretreatment with AM251 blocked the antinociceptive effects of AEA. But, pretreatment with $0.4 \mu \mathrm{g}$ of IRTX partially modulated the antinociceptive effects of AEA. These findings suggest that blockade of CBI receptor and TRPVI receptor play an important role in the peripheral processing of inflammatory pain in the orofacial area.
\end{abstract}

Keywords: Antinociception, Cannabinoid (CB), Inflammatory pain, Nociceptive behavior, TRPVI

\section{Introduction}

The cannabinoid (CB), which is a main ingredient of marijuana, is substance with various pharmacological actions such as pain control and inflammatory response regulation, and synthesizes and secretes the endocannabinoid under physiological or pathological situation. It has been known that most endocannabinoids bind to and act on a G-protein coupled receptor, or act on TRPV1 (transient receptor potential vanilloid), a cation channel activated by capsaicin or heat stimuli. Cannabinoid receptors are largely classified into CB1 receptors ${ }^{1}$. They are found primarily in the central nervous system, and CB2 receptors ${ }^{2}$, which are observed in immune-derived cells. And it has been reported that they are anatomically distributed in the spinal dorsal horn that is involved in the conduction and control of pain. A lot of findings from research using laboratory animals revealed that cannabinoid receptors typically show analgesic action ${ }^{3}$. A cannabinoid receptor agonist injected in the meningeal space alleviated hyperalgesia and allodynia in neuropathic and inflammatory pain models, and D9-tetrahydrocannabinol (THC) and anandamide (AEA), which were injected into the abdominal cavity, reduced pain from excitotoxic pressure on the plantar surface in animal models of arthritis. Recently, it was reported that the topical use of WIN 55,212-2, an agonist of CB1 and CB2, reduced the activation of wide dynamic range (WDR) neurons within the medulla oblongata ${ }^{4}$. These findings demonstrated that the activity of $\mathrm{CB}$ receptors can contribute to pain control in the orofacial area.

A damaged peripheral nerve or tissue sends a signal to the spine cord via primary afferent nerve fibers, and diverse pathways including the TRPV1 channel exist at the endings of primary afferent nerve fibers. A number of experimental models revealed that the TRPV1 channel is also involved in the occurrence and regulation of pain in the orofacial area, which is controlled by the trigeminal nerve; and Zhang et al. ${ }^{6}$ reported that orofacial pain induced by the injection of capsaicin into the whisker area of a laboratory animal can be controlled by inhibiting the activity of the TRPV1 channel. Similarly, it has also been reported that allodynia and thermal hyperalgesia induced by the injection of IL-1 $\beta$ into the orofacial area were significantly reduced by the injection of iodoresiniferatoxin (IRTX), an inhibitor of the TRPV1 channel. Besides, it was demonstrated that inflammatory pain in the temporomandibular joint induced by formalin injection is reduced by capsazeptine, another inhibitor of the TRPV1 channel ${ }^{5}$. The facial myalgia induced by the injection of mustard oil in the masseter muscle area is alleviated by capsazepine. It is deemed that these findings are sufficient to prove the involvement of the TRPV1 channel in various forms of pain conduction occurring in the orofacial area.

The present study induced orofacial pain by injecting formalin in the hypodermic right whisker area of a laboratory animals via a tube intubated into the area, and evaluated the effects of the cannabinoid on pain control by injecting AEA in the same area before formalin injection. 


\section{Methods}

\subsection{Animals and Surgery}

To conduct this study, male Sprague-Dawley white rats $(240-280 \mathrm{~g})$ were used. Under the constant condition of $23-24^{\circ} \mathrm{C}$, the rats were maintained in a 12-h light and 12-h dark cycle, and water and food were provided freely. For 30 minutes before the observation of changes in nociceptive behavior, the laboratory animals were exposed to the experimental environment for their adaptation to the space; and stress to the laboratory animals caused by behavioral suppression, etc. were minimized before the experiment, as far as possible. All behavioral responses were evaluated with different surgeons (for surgery and drug infusion) and evaluators. This study was carried out in compliance with the Pain Research Society's ethical guidelines for the use of experimental animals in biomedical research.

\subsection{Measurement of Formalin-induced Nociceptive Behavior}

$5 \%$ formalin $(30 \mathrm{ul})$ was injected in the hypodermic right whisker area of the laboratory animals through an intubated tube in order to induce orofacial pain in the laboratory animals; their behavior was observed for a total of 45 minutes at an interval of five minutes following the injection; and rubbing or scratching the orofacial area in which the drug was injected was counted as a pain marker. The observation of formalin-induced nociceptive behavior in this study was conducted with the same methods as those in Park et al. $^{7}$

\subsection{Changes in Nociceptive Behavior caused by the Injection of CB1 Receptor Agonist (AEA)}

To experiment the effects of $\mathrm{CB} 1$ receptor agonist injection on orofacial pain induced by formalin injection, AEA was injected in the hypodermic area on the same side 10 minutes before formalin injection. Three days before the drug injection experiment, a polyethylene tube (PE10) for drug injection was introduced into the hypodermic orofacial area of the laboratory animal after the animal was anesthetized with a mixture of Zoletil and Ksilazol. To check the activity of CB1 receptors, AEA $(0.03-0.3 \mu \mathrm{g} / 30 \mu \mathrm{l})$ was injected into the animals after they recovered for 72 hours after the surgery, via polyethylene tubes. AEA was purchased from Calbiochem, and was dissolved in a vehicle of $70 \%$ DMSO and $30 \%$ saline. The control group was evaluated after the injection of a solution of $70 \%$ DMSO and $30 \%$ saline, the vehicle of AEA.

\subsection{Changes in Nociceptive Behavior Induced by Injecting the Inhibitor of CB1 Receptor or TRPV1 Channel}

To experiment the effects of CB1 rector or TRPV1 channel inhibitors, inhibitors were injected 10 minutes before $\mathrm{CB} 1$ reactor agonist injection, via the polyethylene tube intubated in the hypodermic orofacial area on the same side. To inhibit the activity of the CB1 receptor, AM251 (10 $\mu \mathrm{g} / 20 \mu \mathrm{l})$ or IRTX $(0.4 \mu \mathrm{g} / 30 \mu \mathrm{l})$, inhibitors of the CB1 receptor or the TRPV1 channel, was injected in the hypodermic orofacial area. AM251 and IRTX were purchased from Calbiochem, and were dissolved in a vehicle of $70 \%$ DMSO and $30 \%$ saline, respectively. The control group was evaluated after the solvent of the same volume was injected in them.

\subsection{Statistics}

Statistical analysis of changes in behavioral date was carried out with one-way analysis of the variance (ANOVA). In all statistical comparisons, $p<0.05$ was used as the criterion for statistical significance. All date are represented as mean \pm standard error.

\section{Results}

Figure 1 illustrates the number of scratches produced by formalin before the subcutaneous injection of AEA, a CB1 receptor agonist. The subcutaneous injections of the control group affect the number of scratches. $0.003,0.03,0.3 \mu \mathrm{g}$ of AEA injected significant nociceptive effects $(p<0.05)$.

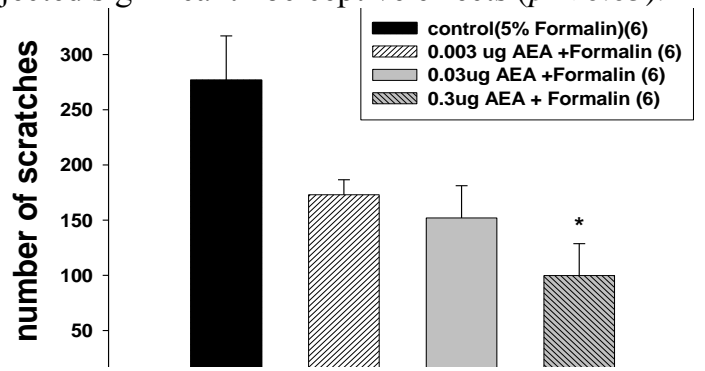

Figure 1: Effects of AEA, CB1 receptor agonist, injected hypodermic area on the number of scratching response produced by formalin. Number of animals was 6 in each group. $P<0.05$, control vs $0.3 \mu \mathrm{g}$

AEA+Formalin 
The change in the number of scratches produced by formalin before the injection of AM251, inhibit the activity of the CB1 receptor, illustrated in Figure 2. $10 \mu \mathrm{g}$ AM251 and $0.3 \mu \mathrm{g}$ of AEA injected significant nociceptive effects.

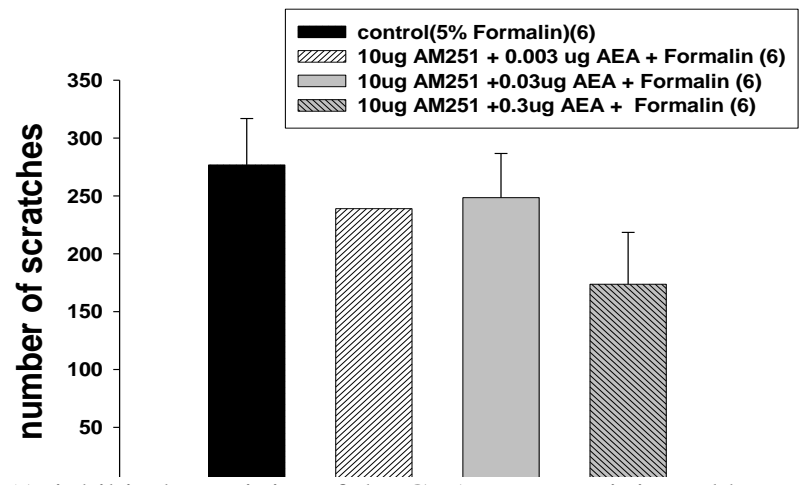

Figure 2: Effects of AM251, inhibit the activity of the CB1 receptor, injected hypodermic area on the number of scratching responses produced by formalin. Number of animals was 6 in each group.

The effects of the AEA, CB1 receptor agonist, IRTX, TRPV1 channel inhibitors on the scratching behavior produced by formalin illustrated in Figure 3. $0.4 \mu \mathrm{g}$ IRTX and $0.3 \mu \mathrm{g}$ of AEA injected significant antinociceptive effects $(p<0.05)$.

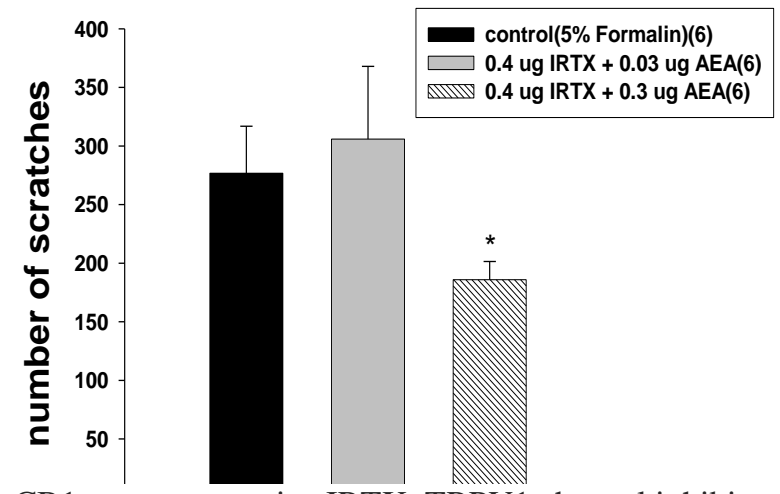

Figure 3: Effects of AEA, CB1 receptor agonist, IRTX, TRPV1 channel inhibitors, injected hypodermic area on the number of scratching responses produced by formalin. Number of animals was 6 in each group. $p<0.05$, control vs $0.4 \mu \mathrm{g}$ IRTX+0.3 $\mu \mathrm{g}$ AEA.

\section{Discussion}

The present study induced orofacial pain by injecting formalin in the hypodermic right whisker area of a laboratory animals via a tube intubated into the area, and evaluated the effects of the cannabinoid on pain control by injecting AEA in the same area before formalin injection ${ }^{8}$. Further, changes in nociceptive behavior were observed by preprocessing each inhibitor in order to evaluate whether the role of AEA in the control of orofacial pain induced by formalin injection is involved in the activation of the CB1 receptor and the TRPV1 channel. Several investigators have reported neurotoxic effects of cannabinoids. For instance, AEA induces apoptosis by activation of $\mathrm{CB}_{1}$ receptor in bone marrow-derived dendritic cells ${ }^{9}$. In addition to these findings, a recent study reported that AEA, an endocannabinoid, acts as a ligand that can activate the TRPV1 channel ${ }^{6}$. Some studies showed that the CB1 receptor and the TRPV1 channel are co-expressed in dorsal root ganglion (DRG) neurons, and reported that the CB1 receptor as well as the TRPV1 channel is observed in A fibers and C fibers, which play major roles in pain conduction. These findings suggest that blockade of CB1 receptor and TRPV1 receptor play an important role in the peripheral processing of inflammatory pain in the orofacial area. Moreover, the present results indicate that a targeted blockade of CB1 receptor and the TRPV1 channel is a potentially important new method of treatments for inflammatory pain in the orofacial area. The discovery that the capacity to detect many forms of noxious stimuli is mediated by high-threshold TRP channels expressed selectively in nociceptors has provided insights into the molecular basis of sensory labelled lines — neural input channels devoted to specific types of stimuli and in the peripheral nervous system ${ }^{10}$. 


\section{References}

[1]. N. Egashira, T. Matsuda, E. Koushi, F. Higashihara, K. Mishima, S. Chidori, Hasebe N, K. Iwasaki, R. Nishimura, R. Oishi, and M. Fujiwara, Delta(9)-tetrahydrocannabinol prolongs the immobility time in the mouse forced swim test: involvement of cannabinoid $\mathrm{CB}$ (1) receptor and serotonergic system, European Journal of Pharmacology, 2008, 28: 117-121.

[2]. E. Muro, T. Pons, N. Lequeux, A. Fragola, N. Sanson, Z. Lenkei, and B. Dubertret, Small and stable sulfobetaine zwitterionic quantum dots for functional live-cell imaging, Journal of American Chemistry Society, 2010, 7: 4556-45577.

[3]. K. Matsuda, Y. Mikami, K. Takeda, S. Fukuyama, S. Egawa, M. Sunamura, I. Maruyama, and S. Matsuno, The cannabinoid 1 receptor antagonist, AM251, prolongs the survival of rats with severe acute pancreatitis, The Tohoku Journal of Experimental Medicine, 2005, 207: 99-107.

[4]. A. Papagianni, E. Kokolina, M. Kalovoulos, A. Vainas, C. Dimitriadis, and D. Memmos, Carotid atherosclerosis is associated with inflammation, malnutrition and intercellular adhesion molecule-1 in patients on continuous ambulatory peritoneal dialysis, Nephrology Dialysis Transplantation, 2004, 5: 1258-1263.

[5]. M.B. Urtado, G.H. Gameiro, C.H. Tambeli, L. Fischer, C.B. Urtado, and M.C. Veiga, Involvement of peripheral TRPV1 in TMJ hyperalgesia induced by ethanol withdrawal, Life Sciences, 2007, 81: 1622-1626.

[6]. X.F. Zhang, P. Han, T.R. Neelands, S. McGaraughty, P. Honore, C.S. Surowy, and D. Zhang, Coexpression and activation of TRPV1 suppress the activity of the KCNQ2/3 channel, The Journal of General Physiology, 2011, 138(3): 341-352.

[7]. M.K. Park, J.H. Lee, G.Y. Yang, K.A. Won, M.J. Kim, Y.Y. Park, Y.C. Bae, and D.K. Ahn, Peripheral administration of NR2 antagonists attenuates orofacial formalin-induced nociceptive behavior in rats, Progress in Neuro-Psychopharmacology \& Biological Psychiatry, 2011, 35: 982-986.

[8]. S. Hunskaar, O.B. Fasmer, and K. Hole, Formalin test in mice, a useful technique for evaluating mild analgesics, Journal of Neuroscience Methods, 1985, 14: 69-76.

[9]. Y. Do, R.J. McKallip, M. Nagarkatti, and P.S. Nagarkatti, Activation through cannabinoid receptors 1 and 2 on dendritic cells triggers $\mathrm{NF}-\mathrm{\kappa B}$ dependent apoptosis: novel role for endogenous and exogenous cannabinoids in immunoregulation, Journal of Immunology, 2004, 173: 2373-2382.

[10]. A. Patapoutian, S. Tate, and C.J. Woolf, Transient receptor potential channels: targeting pain at the source, Nature Reviews Drug Discovery, 2009, 8: 55-68. 